\title{
Considerations in Management for Trans and Gender Diverse Patients with Inherited Cancer Risk
}

\author{
Beth Coad ${ }^{1,2}$ - Josephine Giblin ${ }^{3,4} \cdot$ Reubs J. Walsh $^{5,6,7}$. Oliwia Kuczkowska ${ }^{1}$ Alexander M. von Vaupel-Klein ${ }^{8}$. \\ Alison Berner 9,10
}

Accepted: 11 August 2021 / Published online: 27 September 2021

(c) The Author(s) 2021

\begin{abstract}
Purpose of Review To best support all patients with inherited cancer risk, we must broaden our scope of practice to consider the needs of the transgender and gender diverse (trans) community. We considered best practice for supporting trans patients including tailored risk assessments and management recommendations.

Recent Findings There is limited literature considering trans patient care in cancer genetics. Small case studies have highlighted how medical transition and cancer risk-reducing options intersect with the need for individualised care. Studies have also shown that cancer genetics professionals do not feel prepared to support trans patients.

Summary Patient-centred care for trans patients relies on a multidisciplinary team (MDT) engaged in shared decisionmaking. National guidelines are needed to standardise access to appropriate discussions around risk-reducing options and screening. International collaborative research is required to provide empiric data on the impact of gender-affirming treatments on cancer risk, and more experiential data is needed from trans patients accessing cancer genetics services. Finally, education and training in this area should be formally embedded for all cancer genetics professionals.
\end{abstract}

Keywords Cancer risk $\cdot$ Transgender $\cdot$ Genetics $\cdot$ Personalised care

\section{Introduction}

Across UK cancer genetics services, an increase in referrals for transgender and gender diverse (trans) patients with inherited cancer risk has been observed. This mirrors the rise in demand for Gender Identity Clinic (GIC) services in the UK [1,2].

This article is part of the TopicalCollection on Cancer Genomics

Beth Coad

beth.coad@nhs.net

1 South West Thames Regional Genomics Service, St George's Hospital NHS Foundation Trust, London, UK

2 St George's, University of London, London, UK

3 Wessex Clinical Genetics Service, University Hospital Southampton Foundation Trust, Southampton, UK

4 NHS Central and South Genomic Medicine Service Alliance, London, UK

5 Department of Clinical, Faculty of Behavioural and Movement Sciences, Neuro- and Developmental Psychology, Vrije Universiteit Amsterdam, Amsterdam, Netherlands
A transgender person is someone whose gender is different from their sex assigned at birth. They may have undergone medical transition which can include taking sex-related hormones and gender-affirming surgeries. Gender is not a static binary of male or female, and trans people may identify their gender using other descriptors, for example nonbinary, instead of or in addition to terms typically perceived as binary. Gender is fluid and may change for people over

6 Gender Identity Research and Education Society (GIRES), Ashtead, UK

7 Center for Applied Transgender Studies (CATS), Chicago, IL, USA

8 Faculty of Medicine, Utrecht University Medical Center, Utrecht University, Utrecht, Netherlands

9 Genomics and Computational Biology, Barts Cancer Institute, London, UK

10 Gender Identity Clinic, Tavistock and Portman NHS Foundation Trust, London, UK 
time. Many trans people experience trans gender dysphoria, a specific distress caused by an incongruence between one's gender identity and one's own and/or other people's gendered perceptions of one's body and other sex/gender signifiers $[3 \bullet \bullet]$.

In many clinical specialities, transgender status may not alter the appropriate clinical approach, and the assumption that it does can diminish the quality of care. However, in cancer genetics, trans patients may have different needs from cisgender patients presenting with a similar family history. These needs may arise from the impact of medical transition on cancer risk, or from psychosocial factors associated with genetic testing. These include, but are not limited to, gender dysphoria, challenging family communication, timing of testing and the potential exacerbation of risks by stress.

In fast-changing fields like cancer genetics, and young specialities like gender identity healthcare, ongoing research is crucial in supporting patients appropriately. Sutherland et al. have summarised considerations in hereditary breast and gynaecological cancers, highlighting a common theme: a series of unanswered questions [4•]. Considering transinclusive genetic counselling, some guidance is available around cultural competency, inclusive pedigree symbols and practice recommendations $[3 \bullet \bullet$. There has also been some consideration of the experience of genetic counsellors seeing trans patients $[5,6]$; however, there is minimal literature containing experiences from the patients' perspective beyond one study by Barnes et al. [ $7 \bullet \bullet$. The majority of literature is from a US perspective and does not reflect all aspects of the experience of trans patients in the UK.

Here, we consider the experiences and management of trans patients accessing UK cancer genetics services. We provide a background to gender identity care in the UK and discuss a series of cases highlighting the needs of trans patients with inherited cancer risk, including a direct patient perspective. We outline recommendations for cancer genetics clinicians seeing trans patients, and the clinicians who refer them. Finally, we outline the further work required to improve cancer genetics services for these patients.

\section{Gender Identity Care in the UK}

Patients may choose to access transition-related care through either the NHS or private routes, or a mixture of both.

Within the NHS, there are seven adult Gender Identity Clinics (GICs): two in Scotland and one in Wales. These can be accessed by GP referral. These are staffed by medical doctors and psychologists and make recommendations around suitability for gender-affirming hormonal therapies, surgeries and other interventions and may also offer speech and language therapy and/or counselling psychology [8]. In addition, a number of gender service pilots were announced in England in 2020 [9-12]. Adults currently on the waiting list for a GIC will be able to access hormones, and in some cases, surgical referrals, more rapidly through this route. Some clinics also have further services on-site, such as trans-specific sexual health.

The NHS Gender Identity Development Service (GIDS) assesses children with suspected gender dysphoria and has bases in London and Leeds, as well as outreach clinics in other major cities.

There are currently long waiting lists for NHS GICs and GIDS, where patients can wait over 3 and a half years from referral [13]. There are also long gaps between appointments [14]. Patients may therefore access hormones via the internet to self-medicate $[15,16]$. GPs may or may not provide clinical oversight of this depending on expertise and patient disclosure. GPs can also choose to provide "bridging" hormone prescriptions prior to assessment by a GIC if they feel the psychological and harm-reduction benefits outweigh any risks. However, despite clear guidance [17], many refuse, due to lack of experience or training, fear of litigation or outright transphobia.

A list of gender affirming treatments that may be accessed by trans patients can be found in Table 1 . Note that nonbinary patients, as well as some trans feminine and trans masculine people may choose to access both masculinising and feminizing treatments [18].

\section{Challenges for Trans Patients with Inherited Cancer Risk}

\section{Barriers to Healthcare}

\section{Monitoring}

Currently, NHS healthcare information systems ask for only male or female "gender" at registration. There is no approved Information Standard for gender identity or transgender status monitoring [19], though at the time of writing, this is being developed by LGBT Foundation, CliniQ, Action for transgender Health and National LGB\&T Partnership based on the questions used by the HIV and AIDS Reporting Data Set [20].

This creates a number of barriers to care. Patients are unlikely to want to engage with a service that demonstrates ignorance about their medical history and fails to recognise their identity. This can impair rapport with genetics professionals. Patients may choose not to disclose their transgender status and be given inaccurate advice. In addition, if we fail to collect data on our trans population, we will fail to detect trends in both their clinical outcomes and the quality of their care. 
Table 1 Gender-affirming treatment options available through NHS and private healthcare

\begin{tabular}{|c|c|c|c|}
\hline Effect & Treatment or intervention & Types & NHS funded \\
\hline \multirow[t]{13}{*}{ Feminising } & \multirow[t]{3}{*}{ Oestrogen } & Tablet & \multirow[t]{3}{*}{ Yes } \\
\hline & & Patch & \\
\hline & & Gel & \\
\hline & GnRHa & Injection & Yes \\
\hline & 5-alpha reductase inhibitor & Tablet & No \\
\hline & Progesterone & Tablet & No \\
\hline & \multirow[t]{2}{*}{ Genital reconstructive surgery } & Vaginoplasty & Yes \\
\hline & & Vulvoplasty & $\begin{array}{l}\text { (some other surgery types also } \\
\text { available privately) }\end{array}$ \\
\hline & \multirow[t]{2}{*}{ Facial hair removal } & Laser & \multirow[t]{2}{*}{ Country and region-dependent } \\
\hline & & Electrolysis & \\
\hline & Breast augmentation & Various available & No \\
\hline & Facial feminisation & Various available & No \\
\hline & Cricothyroid shave & $\mathrm{n} / \mathrm{a}$ & No \\
\hline \multirow[t]{7}{*}{ Masculinising } & \multirow[t]{2}{*}{ Testosterone } & Injection & \multirow[t]{2}{*}{ Yes } \\
\hline & & Gel & \\
\hline & GnRHa & Injection & Yes \\
\hline & Bilateral mastectomy and male chest reconstruction & $\mathrm{n} / \mathrm{a}$ & Yes \\
\hline & $\begin{array}{l}\text { Total abdominal hysterectomy and bilateral salpingo- } \\
\text { oophorectomy (TAH and BSO) }\end{array}$ & $\mathrm{n} / \mathrm{a}$ & Yes \\
\hline & \multirow[t]{2}{*}{ Genital reconstructive surgery } & Phalloplasty & \multirow[t]{2}{*}{ Yes } \\
\hline & & Metoidioplasty & \\
\hline
\end{tabular}

\section{Stigma and Microaggressions}

Stonewall [21] reported that $41 \%$ of trans people experienced healthcare staff who lacked understanding of their specific health needs, and 7\% had been refused access to healthcare. Trans patients may be less likely to engage with a range of healthcare services due to fear of discrimination [22]. This is exacerbated in the field of genetics where informational and awareness-raising materials rarely use gender neutral terms, with practices that can be based on cisnormative and heteronormative assumptions [23].

\section{Experiences from the Cancer Genetics and Gender Identity Clinic}

Cancer family histories raise complex issues around individual risk, screening and preventative options, as well as patient choice. No two patient experiences are the same, but shared lessons can be learned from individual stories. The following vignettes have been anonymised or fabricated using a combination of cases from the authors' (BC, $\mathrm{AB}$ and JG) clinical experiences, to highlight the needs of trans patients with a family history of cancer.

\section{Case 1: Dysphoria in the Cancer Genetics Clinic}

\section{Clinical Scenario}

A 28-year-old transgender man was referred to genetics by his GIC at $50 \%$ risk of a familial BRCAl pathogenic variant. He was taking testosterone and considering bilateral mastectomy with male chest reconstruction.

\section{Identified Issues}

- Discussions around breasts/ovaries and dysphoria

- Chest tissue and cancer risk

- Ovarian cancer risk

\section{Recommended Management}

While dysphoria is not experienced by all trans patients, clinicians should be aware of the potential impact of cancer genetics discussions on gender incongruence. Using gender neutral anatomical terms may alleviate this, for example 'chest', when discussing breast cancer with transgender men.

Appropriate screening and surgical options should be discussed with BRCA carriers who were assigned female at birth. Most, but not all, male chest reconstructions leave 
some remaining breast tissue [24], and so would provide less cancer risk reduction. However, complete mastectomy with lipofilling would be a viable alternative. This would be expected to provide risk reduction comparable to cisgender women who undergo bilateral mastectomy with autologous reconstruction.

Not all transgender men will choose to undergo mastectomy [25], and most who do will retain breast tissue. In this case, an MDT approach should be used to work with screening units to consider the most appropriate screening methods that minimise dysphoria. Mammography will not be possible on a patient who has undergone male chest reconstruction, and so, annual MRIs are more appropriate and are less likely to exacerbate dysphoria.

As with chest surgery, choices around total abdominal hysterectomy and bilateral salpingo-oophorectomy (TAH and BSO) vary between patients. Clinicians should avoid assumptions about patients' surgery choices. Instead, shared decision-making [26] should be used to consider which options are best for the patient given their cancer risk, transition and preferences about fertility preservation.

\section{Case 2: The Impact of Hormones on Cancer Risk}

\section{Clinical Scenario}

A 32-year-old non-binary BRCA2 carrier did not express that their gender was incongruent with the male sex they were assigned at birth when taken through predictive testing. After this, they began taking oestrogen ordered online for feminization to minimise dysphoria and were re-referred to genetics by their GIC.

\section{Identified Issues}

- Oestrogen and breast cancer risk

- Prostate cancer risk and associated dysphoria

\section{Recommended Management}

There is a lack of data regarding the impact of oestrogen on breast cancer risk in trans patients. De Blok et al. compared breast cancer cases in transgender women taking oestrogen and cisgender men [27]. They found a 47-fold increase in breast cancer incidence and a younger age of onset, though still 3 times less than the incidence in cisgender women. This study included trans women who had taken oestrogen across a range of time frames and did not include information on cancer family history for most patients, except one known BRCAl carrier. More data are needed to elucidate effects of oestrogen on breast cancer risks in those with a family history.
In transgender women, oestrogen is typically dosed to achieve levels in pubertal female range lifelong [28, 29]. Therefore, breast cancer risk in trans patients may be reduced by lowering oestrogen to a maintenance dose after 5 years, the average timeframe to achieve the desired feminisation. A non-binary person may also wish to do this or may choose a lower dose throughout depending on the degree of feminisation they wish to achieve.

Clinicians may be uncomfortable knowing the patient is self-medicating with oestrogen. Clinicians should employ a patient-centred approach acknowledging how both continuing or stopping hormones could have health implications either upon cancer risk, or risk of dysphoria and/or harassment and resultant stress-related mental and physical health problems. In this situation, the genetics service could support a patient to seek a bridging prescription from their GP.

Transgender patients assigned male at birth who are $B R C A 2$ carriers will not have their prostate removed if they undergo genital surgery so these patients should be offered prostate screening [30]. The patient should be informed of the screening process so they can prepare for this, and an MDT approach should be taken to minimize the impact of dysphoria. It may be appropriate to refer to specialist clinics with experience of prostate cancer screening in transgender patients, as a digital prostate exam may be more challenging due to atrophy.

\section{Case 3: Timing of Genetic Testing}

\section{Clinical Scenario}

A 20-year-old transgender woman was referred to genetics at $50 \%$ risk of a familial BRCA2 pathogenic variant. At this time, predictive testing was secondary to her desire to begin hormone treatment. After thorough discussion, she decided not to pursue predictive testing and felt she may consider this closer to age 30 . In the following year, she began hormone therapy. However, within a few months, she re-contacted the genetics service distressed. She stated that the GIC wished to know her BRCA2 status before increasing her dose of oestrogen. She was then seen for further discussion in the genetics clinic, and again chose to delay her predictive testing. In addition to being several years from the age of risk significantly increasing, she reflected on how she might struggle to cope with a result at this stage of her life, being relatively early within her transition, a university student and experiencing the fallout of breast cancer and $B R C A 2$ testing in her family.

\section{Identified Issues}

- Breast and prostate cancer risks

- Timing of risk-reducing surgery and hormone treatment 
- Inherited cancer risk as a barrier to accessing hormones

- Lack of communication between genetics services and a GIC

\section{Recommended Management}

As discussed above, providing this patient with an accurate breast cancer risk assessment following gender-affirming hormone treatment is difficult, with her risk of prostate cancer similar to cis male BRCA2 carriers. Here, as above, all surgery and risk-reducing options should be discussed with the patient during their genetics appointment.

There are currently no formal guidelines for surgical management of transgender BRCA carriers. This case raises a potential argument for risk-reducing mastectomy and reconstruction prior to starting hormone therapy in appropriate circumstances. Such an approach has the benefit of negating much of the cancer risk related to breast growth on oestrogen while allowing the patient to experience its other effects. Breast reconstruction in itself may reduce dysphoria. This option was not discussed with the patient as the genetic service had not connected with the local GIC to consider this, highlighting the importance of an MDT approach.

The choice to pursue predictive testing is complex for many young adults [31], irrespective of the psychosocial complexities of gender transition. Real or perceived pressure from a GIC can lead to patients undergoing genetic testing against their true wishes, for fear of losing access to gender affirming treatment [6]. Similarly, declining to undergo genetic testing might be used, wholly inappropriately, as a barrier to treatment, as described above. It is unethical for genetic testing to be a prerequisite, but it is important that patients have all the relevant information about risks and benefits before starting gender affirming treatment [32]. In cases such as this, (and where the risk of a variant is identified by the GIC) a suitable approach may be a referral to clinical genetics for a dedicated discussion.

\section{Case 4: It is Not All About BRCA}

\section{Clinical Scenario}

A 40-year-old transgender woman was seen in the GIC. She had a history of confirmed familial adenomatous polyposis (FAP) caused by a pathogenic $A P C$ variant. She had undergone prophylactic colectomy and had developed an abdominal wall desmoid tumour. She wished to access gender-affirming hormones and genital surgery. She also wished to discuss her options for fertility preservation as she had not wanted to pass on her condition and was aware she may be eligible for pre-implantation diagnosis (PGD).

\section{Identified Issues}

- Inherited cancer risk and gender-affirming treatment interactions outside of BRCA

- Desmoid tumour risk from oestrogen and gender affirming surgery

- Reproductive considerations

- Requirements for MDT approach

\section{Recommended Management}

One in five with FAP develop desmoid tumours [33]. Their growth can be driven by oestrogen, and in cisgender populations, anti-oestrogen therapy may be effective in up to $50 \%$ of cases [34]. The GIC clinician should seek advice from specialists including cancer geneticists, gastroenterologists or surgeons working in FAP and their local endocrinologist in order to gather the information to appropriately discuss risks and benefits of oestrogen with the patient. It may be useful to conduct a mini-MDT meeting or involve the other clinicians in a single patient consultation.

If the patient is happy to accept the risk of a growing desmoid, then they should be commenced at the lowest dose of oestrogen (as is usual practice) and monitored with regular imaging as per the specialists' recommendations [28]. Any increase in dose will be a joint decision between the patient and the GIC considering blood results and monitoring imaging. It is important to acknowledge the uncertainty here given the lack of research. One strategy, as in the $B R C A 2$ case above, might be to aim for female range oestradiol levels for the first 5 years to enable anatomical changes and then to consider lowering the dose of oestrogen, to reduce the risk of further desmoid development.

In patients without a desmoid, the risks of development would still need to be discussed and regular imaging undertaken.

As desmoids tend to develop at sites of previous surgery, genital reconstruction surgery also presents additional risks and may not achieve the desired cosmetic outcome. Importantly, the diagnosis of FAP with or without desmoid tumours should not prevent a patient receiving a referral, but the surgeon should be made aware of the history. Previous colectomy or current desmoids may make surgeries, in particular vaginoplasty, too technically difficult, and this is a surgical decision.

Regardless of gender identity, sexual orientation or any diagnosis, one should not make assumptions about future wishes for biological children. This patient is eligible for PGD in England, since FAP is on the Human Fertilisation and Embryology Authority eligibility list [35]. Genderaffirming hormone regimens typically suppress fertility, and while this suppression is reversible in a proportion of individuals (by stopping the hormone treatment), it is not yet 
possible to make any predictions in individual cases [36]. Trans patients may also access surgeries that remove the gonads, causing irreversible infertility. They are therefore eligible for gamete storage under the HFEA guidelines [37]. This should be offered prior to commencing hormones but remains an option later, though the patient may struggle with dysphoria from stopping hormones. It should be noted that eligibility for NHS funding for storage and fertility treatments varies by devolved nation, and by clinical commissioning group in England.

Finally, a diagnosis of FAP necessitates interaction with other specialities and departments, e.g. imaging and endoscopy, who may not have the same level of training and cultural competency. Genetics clinicians should explore how the patient found their interactions with these departments as they are unlikely to want to engage with the recommended screening if they are met with discrimination or a lack of consideration. It may be necessary to contact the departments in question to provide education or indeed to raise concerns if needed.

\section{Case 5: The Need for Tissue-Specific Guidance}

\section{Clinical Scenario}

Figure 1 pedigree showing a 45-year-old transgender woman who was referred from the breast cancer family history clinic where both herself and her 42-year-old cousin were seen. No genetic testing was carried out in the family, and they were not eligible for NHS testing. She had been on oestrogen for the last 5 years and had GRS a year ago.

\section{Identified Issues}

- Considering risk in families without a known inherited cancer risk syndrome

- National screening guidance is not inclusive of trans patients

\section{Recommended Management}

Patients with a family history of breast cancer with no known inherited cause are currently recommended breast screening based on NICE CG164 guidance, which does not clarify considerations for trans patients [38].

Basing screening guidance on a person's individual cancer risk in (a) specific tissue/s would be more inclusive for trans patients and increase equity in the quality of care patients receive. However, more data are needed to adequately assess lifetime cancer risks in trans patients with an inherited risk. Until such data are available patients should be counselled on a case-by-case basis, supporting informed decision-making around appropriate screening

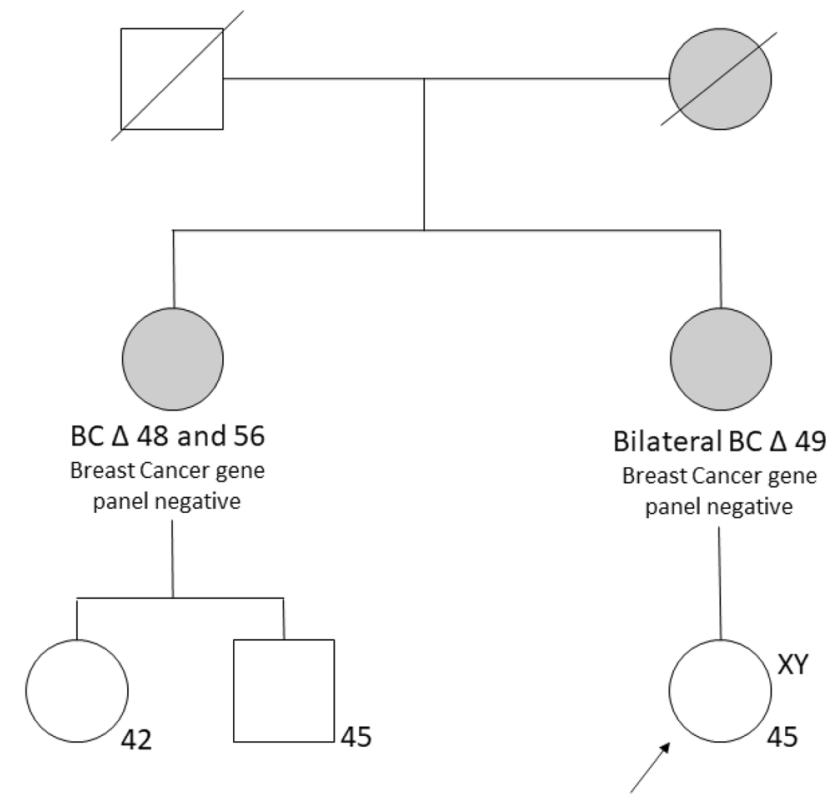

Fig. 1 Pedigree showing a 45-year-old transgender woman who was referred from the breast cancer family history clinic where both herself and her 42-year-old cousin were seen. She has been on oestrogen for the last 5 years and has had GRS.

for each individual. Transgender women, like the above patient (Fig. 1), should be offered the same breast screening as cisgender women with an equivalent family history. However, they should be informed that their lifetime risks will likely be lower, given available data [27].

\section{Case 6: The Impact of Transition on Family Systems}

\section{Clinical Scenario}

A 63-year-old woman was referred to genetics following an endometrial cancer diagnosis and was found to carry an MLH1 pathogenic variant which causes Lynch syndrome. This is an inherited cause of bowel, endometrial, ovarian and some other cancers. In clinic, the patient explained she had a 40-year-old child who had been assigned female at birth and was in the process of transitioning. The patient used a range of pronouns and different names for their child, and expressed discomfort with their transition, explaining that consequently, they were no longer in regular contact.

\section{Identified Issues}

- Endometrial and ovarian cancer risks in Lynch syndrome

- Potential impact of transition on family communications 


\section{Recommended Management}

As with previous cases, there are some transition-specific cancer risks to consider in Lynch syndrome. If a trans person is taking testosterone, there are concerns that this may increase endometrial cancer risk due to endometrial thickening [27] though evidence is conflicting. It is therefore important to discuss risk-reducing TAH and BSO if this had not already been performed as part of gender affirming surgery.

Challenging family relationships are not unusual in cancer genetics, and breakdowns in family communications are reported for a variety of reasons. This case highlights the impact of familial support and rejection of trans people [39, 40]. Standard practice, such as "dear relatives" letters passed through mutual connections, can support communication, and ethics committee discussions may be relevant where this is not possible. It is also important to consider that a patient may not have disclosed their transgender status to their family, and so, as is common when working with families in genetics, confidentiality is key.

Trans patients, and especially those who have experienced familial rejection, may have a 'chosen family'. These provide a crucial support network and should be considered when assessing patients' support through their genetic testing process (Box 1).

\section{Box 1: The patient perspective}

"There was a lack of mental support at the early stages of transition when I was coping with this and my genetic diagnosis. I was offered psychological support, however due to a long waiting list with a waiting time of 3 years the biggest problems of my transition had to be solved alone so I could start living a normal life.

Another big issue was my partner was not offered any psychological support. Patient's partners must deal with complete changeover of their feelings. In some ways they must grieve their partner in order to be able to live with a new one - their transgender partner. Due to my genetic condition, my partner and I were entitled to PGD which we had started to undertake. My transition took a toll on my partnership and it did not survive. Although we are still very close we are no longer a couple and PGD treatment was cancelled.

There is often a lack of communication between doctors and the GIC. I was constantly advised to speak to my GIC clinicians directly, which felt odd as my assumption was that the GP would have quicker access to a relevant person from the gender clinic than myself. Since I have other health issues, I was afraid that a lack of adequate hormonal treatment could lead to serious damage to my body, causing my dysphoria to be experienced more severely. Fortunately, my leading doctor linked my case with an oncologist who also specialised in gender identity problems.

I strongly believe that establishing the MDT would help these issues and many people in a similar situation would not have to go through the same rocky path as I have had to go through."

\section{Good Practice for Cancer Genetics Clinicians}

The complex intersection of medical, cultural, ethical and psychosocial aspects of care is present for all patients taken through genetic testing. It is the role of the cancer genetics clinician to develop their cultural competencies to meet the needs of their patients. Von Vaupel-Klein and Walsh have provided a detailed account of cultural competency considerations for genetic counsellors seeing transgender patients that should be used alongside the guidance here $[3 \bullet \bullet]$.

The genetics clinic appointment should provide a space where patients feel comfortable to talk about their gender identity and disclose information about their transitioning process. Clinicians should remember that all patients' choices around gender differ. Figure 2 shows an infographic summary of key considerations for good practice in cancer genetics.

\section{Good Practice for Clinicians Referring to Cancer Genetics}

Clinicians who are likely to refer trans patients to clinical genetics include family history nurses, GPs, gender identity specialists, oncologists and possibly other members of the cancer care team. They may encounter patients at various stages of their transition, which might have different implications for their interaction with the patient, and the immediacy of their referral. In Fig. 3, we provide an infographic summary of key considerations for referral to cancer genetics.

\section{Next Steps}

Several steps are needed for genetics services to provide an equally high standard of care for all patients, regardless of gender modality [41]. There is a stark need for further research across many areas to make accurate risk assessments and provide appropriate recommendations. Robust data are needed on the impact of medical transition and socioeconomic risk factors on cancer risks for trans patients with inherited cancer predisposition. Furthermore, it is important that the experiences of trans patients seen by cancer genetics services are collected and reviewed to identify 
Fig. 2 Good practice guidance for cancer genetics clinicians

\section{TIPS FOR GENETICS CLINICIANS SEEING TRANS PATIENTS}

\section{PERSON CENTRED CARE}
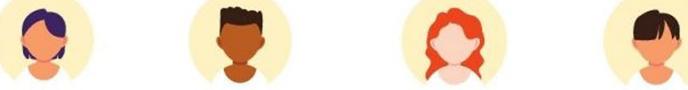

Trans patients should receive the same high standard of person centred care,

free from judgement and assumptions, as every other patient

There are a number of things clinicians can do before seeing trans patients:

Use inclusive family history and patient information forms

Connect with the patients gender identity clinic (GIC) team

Familiarise yourself with aspects of transition mentioned in the referral

\section{PEDIGREE SYMBOLS}

No formal UK guidelines are currently available.

Consider using the symbol that denotes a patients' gender identity and an annotation to show sex assigned at birth. If abbreviations are used, ensure they are clearly defined in a key. e.g.

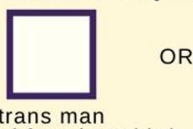

assigned female at birth

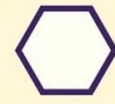

$A M A B$
Key.

$\mathrm{NB}=$ non-binary

$A M A B=$ assigned male at birth

\section{INCLUSIVE LANGUAGE}

Ask patients which pronouns they use (i.e. she/her, he/him, they/their, ze/zir)

Consider gender neutral terms such as "people with ovaries"

Avoid heteronormative assumptions when discussing family planning. Try phrases such as "if you and your partner want children in the future"

Take the lead from the patient and mirror the language they use

Think about non-verbal cues to show inclusivity, such as wearing an NHS rainbow badge and including your pronouns in email signatures

If you make a mistake (i.e. using incorrect pronouns or gendered terms), apologise, correct yourself, and move on

Normalise correcting colleagues' use of language. For example, if a patient is misgendered in a case discussion, point this out briefly and encourage the building of good habits
TISSUE SPECIFIC GUIDANCE

Discuss risk reducing surgery and screening recommendations based upon known increased cancer risks for a specific condition, not assumptions of the tissues patients have

Lynch syndrome patients with ovaries and/or a womb should consider appropriate risk reducing surgery

BRCA carriers with ovaries should considered appropriate risk reducing surgery. Those with a prostate should consider screening. Carriers with breasts/chests should consider screening and risk reducing surgery options

RAD51C/RAD51D/BRIP1 carriers with ovaries should consider appropriate risk reducing surgery

PALB2/CHEK2/ATM carriers with breasts/chests should consider screening and risk reducing surgery options

When assessing risk and discussing screening and surgery, consider the potential impact that any hormone treatment may have on cancer risks

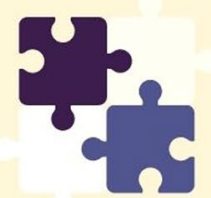

\section{MDT APPROACH}

Seek patient permission to get input from the patient's wider team who may help provide holistic care. This could include clinicians from the GIC, oncology, psychologists or counsellors, GPs, fertility or PGD specialists, and the wider genetics community 
Fig. 3 Good practice guidance for clinicians referring to cancer genetics

\section{TIPS FOR CLINICIANS REFERRING TRANS PATIENTS TO CANCER GENETICS}

\section{REFERRAL LETTERS}

Use patient's name and pronouns unless specifically requested otherwise Ask permission to disclose trans status:

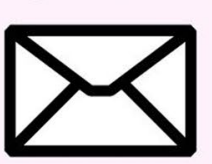

- if permission is given, include information of gender-affirming treatment (current and planned hormones and surgeries) - if permission is denied, patient can choose to be referred as affirmed gender or sex assigned at birth, but should be made aware that genetics may not be able to give accurate information if trans status is not disclosed

\section{GENERAL PRACTITIONERS}

When referring to GICs take a brief family history including 1 st and 2 nd degree relatives with cancer, their age of diagnosis, and type of cancer (particulary breast, ovary, pancreas, prostate, womb and bowel)

If the family history meets criteria for referral to genetics, discuss this with the patient, noting: - some inherited cancer predispositions pose additional risk if patient is on gender-affirming hormones

- inherited cancer predisposition might impact which gender-affirming treatments they wish to access and the risks involved

genetic testing is not mandatory and may not be indicated, but discussion of risks with a genetics specialist is recommended

inherited cancer predisposition will not prevent access to hormones

referrals should be marked as urgent to avoid

delay to gender-affirming treatments

Include family history in referral to GIC and note that patient has been referred to genetics. Include any inherited cancer predisposition that has been confirmed or is being investigated

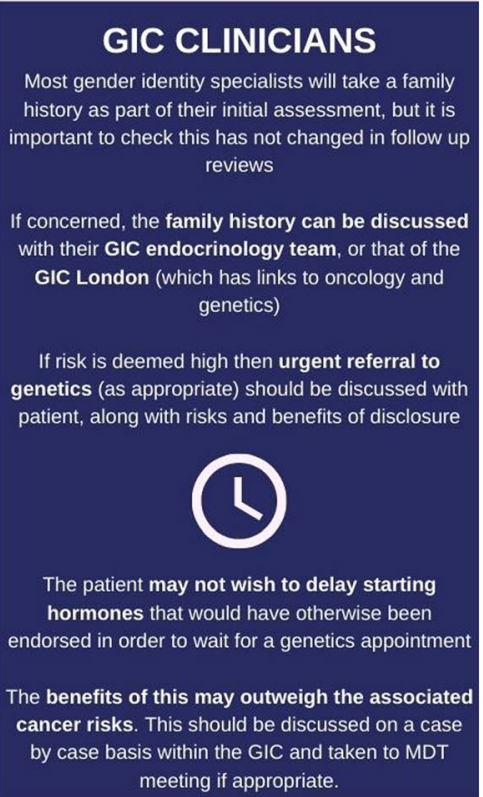

\section{ONCOLOGISTS}

Referrals from oncology should remain part of standard symptomatic testing pathways for which there are local and national guidelines

Literature aimed at educating cancer professionals on the care of trans patients has been published:

Berner, A.M. et al (2021). Education to Improve Cancer Care for LGBTQ+ Patients in the UK. Clinical oncology (R Coll Radiol), 33(4), 270-273.

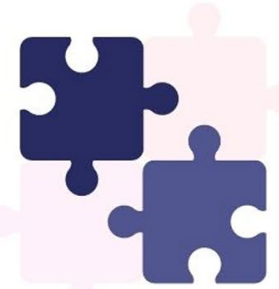

\section{MDT APPROACH}

Given the need to interpret and extrapolate evidence from cisgender populations, there is uncertainty around management of trans patients. An MDT meeting may be useful, including - clinical genetics

- gender identity professionals - oncology team (where indicated)

This can speed up decision making and ensure a coherent plan for the patient. Consider using remote consultant software to facilitate an appointment with multiple professionals simultaneously

\section{OTHER RESOURCES}

www.gmc-uk.org/ethical-guidance/ethical-hub/trans-healthcare www.genomicseducation.hee.nhs.uk/taking-and-drawing-a-family-history/

www.ukcgg.org/information-education

https://play.google.com/store/apps/details?id=com.ubqo.cancergenetics\&hl=en\&gl=US 
areas for service improvement. Similarly, the attitudes and experiences of genetics professionals in the UK should be examined. Such research is necessary to identify and remove barriers to genetics services, and to create a safe and comfortable environment for trans patients.

Studies have shown that more than $80 \%$ of genetic counselling and medical students did not receive training on trans health issues $[6,42]$. These studies are limited by small sample sizes [42] and lack of focus on UK genetic counselling curricula [6]. However, these figures are alarming, especially the finding that only $8 \%$ of UK oncologists felt confident in their knowledge of the specific healthcare needs of LGBT + cancer patients (43). To equip future clinicians with the knowledge and skills to ensure equally high standards of care, it is imperative that their curricula adequately cover topics relevant to trans patients, including, but not limited to, cultural competencies. To ensure professionals maintain these skills, ongoing training for clinicians is required.

Beyond this, reviews of current services are needed to guide the implementation of improvement and national guidance to ensure genetics services are unbiased and accessible, and practice is inclusive. Such wide-scale work is most likely to succeed with international collaboration and support from national organisations, such as the UK Cancer Genetics Group and Genomic Medicine Service Alliances, and through the formation of a national MDT for trans patients with cancer and inherited cancer risk.

\section{Conclusion}

In conclusion, the provision of high-quality equitable care for trans patients with inherited cancer risk will require a personalised approach of genetics and non-genetics clinicians alike. It is only by learning from each other, and foremost from our patients, that we can better meet their needs.

Funding Beth Coad declares some funding from Cancer Research UK through the CanGene-CanVar research programme for an Honorary Clinical Lecturer Salary. Josephine Giblin declares some funding from the NHS Central and South GMSA for a transformation project surrounding care needs of transgender patients. Reubs J Walsh declares a role as Trustee and Treasurer for the Gender Identity Research and Education Society (GIRES), as well as some funding for talks and academic work from NHS Wales, the Association of Genetic Nurses and Counsellors, Prof. Marianne Legato and GIRES. Dr Alison Berner declares some funding from Cancer Research UK for a Clinical PhD fellowship Salary and some funding from Pfizer Genomics for speaking engagements.

\section{Compliance with Ethical Standards}

Conflict of Interest Oliwia Kuczkowska and Alexander M von VaupelKlein have no conflicts of interest to declare.
Human and Animal Rights This article does not contain any studies with human or animal subjects performed by any of the authors.

Open Access This article is licensed under a Creative Commons Attribution 4.0 International License, which permits use, sharing, adaptation, distribution and reproduction in any medium or format, as long as you give appropriate credit to the original author(s) and the source, provide a link to the Creative Commons licence, and indicate if changes were made. The images or other third party material in this article are included in the article's Creative Commons licence, unless indicated otherwise in a credit line to the material. If material is not included in the article's Creative Commons licence and your intended use is not permitted by statutory regulation or exceeds the permitted use, you will need to obtain permission directly from the copyright holder. To view a copy of this licence, visit http://creativecommons.org/licenses/by/4.0/.

\section{References}

\section{Papers of particular interest, published recently, have been highlighted as: \\ - Of importance \\ $\bullet$ Of major importance}

1. Kaltiala R, Bergman H, Carmichael P, de Graaf NM, Egebjerg Rischel K, Frisén L, et al. Time trends in referrals to child and adolescent gender identity services: a study in four Nordic countries and in the UK. Nord J Psychiatry. 2020;74(1):40-4. https:// doi.org/10.1080/08039488.2019.1667429.

2. Fielding J, Bass C. Individuals seeking gender reassignment: marked increase in demand for services. BJPsych Bull. 2018;42(5):206-10. https://doi.org/10.1192/bjb.2018.30.

$3 . \bullet \bullet v o n$ Vaupel-Klein AM, Walsh RJ. Considerations in genetic counseling of transgender patients: Cultural competencies and altered disease risk profiles. J Genet Couns. 2021;30(1):98-109. https:// doi.org/10.1002/jgc4.1372. (This provides useful definitions and cultural competencies guidance for all genetics clinicians.)

4. Sutherland N, Espinel W, Grotzke M, Colonna S. Unanswered Questions: Hereditary breast and gynecological cancer risk assessment in transgender adolescents and young adults. J Genet Couns. 2020;29(4):625-33. https://doi.org/10.1002/jgc4.1278. (This highlights key cases and future research needs in hereditary breast and gynaecological cancers.)

5. Ruderman M, Berro T, Torrey Sosa L, Zayhowski K. Genetic counselors' experiences with transgender individuals in prenatal and preconception settings. J Genet Couns. 2021;00:1-14. https:// doi.org/10.1002/jgc4.1394.

6. Zayhowski K, Park J, Boehmer U, Gabriel C, Berro T, Campion M. Cancer genetic counselors' experiences with transgender patients: a qualitative study. J Genet Couns. 2019;28(3):641-53. https://doi.org/10.1002/jgc4.1092.

7.•Barnes H, Morris E, Austin J. Trans-inclusive genetic counseling services: recommendations from members of the transgender and non-binary community. J Genet Couns. 2020;29(3):423-34. https://doi.org/10.1002/jgc4.1187. (This paper considers the thoughts of the transgender community providing suggestions on inclusive pedigree drawing and validating gender identity.)

8. Richard C, Barrett J. Transgender and non-binary gender healthcare for psychiatrists, psychologists, and other health professionals. Cambridge: Cambridge University Press; 2020. 
9. NHS England. How to find an NHS gender dysphoria clinic - NHS 2020 [Available from: https://www.nhs.uk/live-well/healthy-body/how-tofind-an-nhs-gender-identity-clinic/]. Accessed 24 March 2021.

10. NHS Engalnd. Specialised gender identity services for adults; Report on outcome of public consultation and update to Equality Impact Assessment. NHS England; 2018.

11. 56 Dean Street. Transgender and Non Binary 2020 [Available from: https://dean.st/trans-non-binary/]. Accessed 24 March 2021.

12. LGBT Foundation. LGBT Foundation - gtd healthcare and LGBT Foundation to Launch New Adult transgender Health Service in Greater Manchester 2020 [Available from: https://lgbt.foundation/ news/gtd-healthcare-and-lgbt-foundation-to-launch-new-adulttransgender-health-service-in-greater-manchester/389]. Accessed 24 March 2021.

13. Gender Identity Clinic. Waiting times 2021 [Available from: https://gic.nhs.uk/appointments/waiting-times/]. Accessed 28 April 2021.

14. Vincent B. Transgendergender health: a practitioner's guide to binary and non-binary transgender Patient Care. London: Jessica Kingsley Publishers; 2018.

15. BBC. Transgender patients self-medicating over NHS waits 2020. [Available from: https://www.bbc.co.uk/news/health-51530274]. Accessed 28 March 2021.

16. Taylor J, Zalewska A, Gates JJ, Millon G. An exploration of the lived experiences of non-binary individuals who have presented at a gender identity clinic in the United Kingdom. Int J Transgend. 2019;20(2-3):195-204.

17. GMC. General Medical Council Ethical Guidance Trans Healthcare 2018 [Available from: https://www.gmc-uk.org/ethical-guidance/ ethical-hub/trans-healthcare]. Accessed 28 March 2021.

18. Cocchetti C, Ristori J, Romani A, Maggi M, Fisher AD. Hormonal treatment strategies tailored to non-binary transgender individuals. J Clin Med. 2020:9(6). https://doi.org/10.3390/jcm9061609.

19. NHS England. About The Information Standard: NHS England; 2020 [Available from: https://www.england.nhs.uk/tis/about/]. Accessed 28 March 2021.

20. NHS Digital. NHS Data Model and Dictionary I HIV and AIDS Reporting Data Set 2020 [Available from: https://www.datadictionary. nhs.uk/data_sets/clinical_data_sets/hiv_and_aids_reporting_data_set. html]. Accessed 28 March 2021.

21. Bachmann CL, Gooch B. LGBT in Britain Trans Report. Stonewall and YouGov; 2018.

22. Bachmann CL, Gooch B. LGBT in Britain Health Report. Stonewall and YouGov; 2018.

23. Walser SA, Werner-Lin A, Mueller R, Miller VA, Biswas S, Bernhardt BA. How do providers discuss the results of pediatric exome sequencing with families? Per Med. 2017;14(5):409-22. https://doi.org/10. 2217/pme-2017-0015.

24. Ammari T, Sluiter EC, Gast K, Kuzon WM. Female-to-male gender-affirming chest reconstruction surgery. Aesthet Surg J. 2019;39(2):150-63. https://doi.org/10.1093/asj/sjy098.

25. James SE, Herman JL, Rankin S, Keisling M, Mottet L, Anafi M. The report of the 2015 U.S. transgender survey. Washington, DC: National Center for Transgender Equality; 2015.

26. Elwyn G, Laitner S, Coulter A, Walker E, Watson P, Thomson R. Implementing shared decision making in the NHS. BMJ. 2010;341:5146. https://doi.org/10.1136/bmj.c5146.

27. de Blok CJM, Wiepjes CM, Nota NM, van Engelen K, Adank MA, Dreijerink KMA, et al. Breast cancer risk in transgender people receiving hormone treatment: nationwide cohort study in the Netherlands. BMJ. 2019;365:1652. https://doi.org/10.1136/ bmj.11652.

28. WPATH. Standards of Care for the Health of Transsexual, Transgender, and gender nonconforming people. The World Professional Association for Transgender Health; 2011.
29. RSPsych. Good practice guidelines for the assessment and treatment of adults withgender dysphoria. College Report CR181. London: Royal College of Psychiatrists; 2013.

30. Prostate Cancer UK. Trans women and prostate cancer 2020 [Available from: https://prostatecanceruk.org/prostate-information/ are-you-at-risk/trans-women-and-prostate-cancer.] Accessed 28 March 2021.

31. Godino L, Jackson L, Turchetti D, Hennessy C, Skirton H. Decision making and experiences of young adults undergoing presymptomatic genetic testing for familial cancer: a longitudinal grounded theory study. Eur J Hum Genet. 2018;26(1):44-53. https://doi.org/10.1038/s41431-017-0030-1.

32. Ashley F. Surgical informed consent and recognizing a perioperative duty to disclose in transgender health care. McGill Journal of Law and Health. 2020:13(2).

33. Heiskanen I, Järvinen HJ. Occurrence of desmoid tumours in familial adenomatous polyposis and results of treatment. Int J Colorectal Dis. 1996;11(4):157-62.

34. Calvert GT, Monument MJ, Burt RW, Jones KB, Randall RL. Extra-abdominal desmoid tumors associated with familial adenomatous polyposis. Sarcoma. 2012;2012: 726537. https://doi.org/ $10.1007 / \mathrm{s} 003840050034$.

35. HFEA. PGD conditions 2021 [Available from: https://www.hfea. gov.uk/pgd-conditions/]. Accessed 28 March 2021.

36. Cheng PJ, Pastuszak AW, Myers JB, Goodwin IA, Hotaling JM. Fertility concerns of the transgender patient. Transl Androl Urol. 2019;8(3):209-18. https://doi.org/10.21037/tau.2019.05.09.

37. HFEA. Information for trans and non-binary people seeking fertility treatment 2021 [Available from: https://www.hfea.gov.uk/treatments/ fertility-preservation/information-for-trans-and-non-binary-peopleseeking-fertility-treatment/ ]. Accessed 28 March 2021.

38. NICE. Familial breast cancer: classification, care and managing breast cancer and related risks in people with a family history of breast cancer Clinical Guidance CG164. National Institute for Health and Care Excellence (NICE); 2019.

39. Brown C, Porta CM, Eisenberg ME, McMorris BJ, Sieving RE. Family relationships and the health and well-being of transgender and gender-diverse youth: a critical review. LGBT Health. 2020;7(8):407-19. https://doi.org/10.1089/lgbt.2019.0200.

40. Andrzejewski J, Pampati S, Steiner RJ, Boyce L, Johns MM. Perspectives of transgender youth on parental support: qualitative findings from the resilience and transgender youth study. Health Educ Behav. 2021;48(1):74-81. https://doi.org/10.1177/ 1090198120965504.

41. Ashley F. 'Trans' is my gender modality: a modest terminological proposal. In: Erickson-Schroth L, editor. Trans Bodies, Trans Selves: A Resource for the Transgender Community 2nd Edition ed: Oxford University Publishers; 2021.

42. Parameshwaran V, Cockbain BC, Hillyard M, Price JR. Is the lack of specific lesbian, gay, bisexual, transgender and queer/questioning (LGBTQ) health care education in medical school a cause for concern? Evidence from a survey of knowledge and practice among UK medical students. J Homosex. 2017;64(3):367-81. https://doi.org/10.1080/00918369.2016.1190218.

43. Berner AM, Hughes DJ, Tharmalingam H, Baker T, Heyworth B, Banerjee S, et al. An evaluation of self-perceived knowledge, attitudes and behaviours of UK oncologists about LGBTQ+ patients with cancer. ESMO Open. 2020;5(6): e000906. https://doi.org/10. 1136/esmoopen-2020-000906.

Publisher's Note Springer Nature remains neutral with regard to jurisdictional claims in published maps and institutional affiliations. 